\title{
Analysis of parameters of air passing through the rain zone in a cross- flow
}

\author{
Lukáš Dvořák ${ }^{1, a}$, Jan Čížek ${ }^{1}$ and Jiří Nožička ${ }^{1}$ \\ ${ }^{1}$ CTU in Prague, Faculty of Mechanical Engineering, Department of Fluid Dynamics and Thermodynamics, Technická 4, \\ Praha 6 - Dejvice, 16607
}

\begin{abstract}
The research in the field of cooling towers shows that a rigorous determination of each parameter of air passing through areas with water drops is increasingly important. The transfer of heat, mass and momentum is represented, on the side of the air, as temperature and humidity increase and static pressure decrease due to the interaction between the flowing air and falling drops. The present article focuses on the description of the experimental setup allowing the measurement of these parameters on both the air and the water side, and possible ways to analyze measured values.
\end{abstract}

\section{Introduction}

One of the most important parts of cooling tower, considering the transfer of momentum, heat and mass, is the so-called "rain zone", which is located beneath cooling fill in standard counterflow cooling towers. Water bodies fall out of the cooling fill, whether film- or grid-type, in the form of drops in the air, while the proportion of heat removed from water is not negligible and, for example, D. G. Kröger [1] states that up to $20 \%$ of the total heat removed by the tower fall on the rain zone. Therefore, knowledge and exact mathematical description of the processes, which happen in the rain zone, are important in terms of design of such undoubtedly necessary devices.

For correct design of mathematical model, it is always necessary to verify it by means of one or more experimental models. Given to relatively small changes in temperature and water content in the air, this experimental model must be designed as to maximise values of these changes. Therefore, an experimental stand was needed to verify the mathematical model situated in a vertical position so that both fluids - i.e. cooled and cooling, flow towards each other in almost crossflow way, which corresponds, for example, to air intake at the inlet of the cooling tower with natural draft. Due to crossflow connection, it is not possible and appropriate to use the most frequently used Chebyshev integrations for determining the Merkel number that is dimensionless characteristic of heat and mass transfer. Therefore, the Effectiveness - NTU (Number of Transfer Units) method that may be also applied to evaporative cooling systems [2] is used in this paper.

\section{Analysis}

As mentioned above, the interaction between liquid and gaseous phases happen in the rain zone. Due to temperature and concentration gradient between the two phases, heat is removed from cooled medium, i.e. water, and transferred to cooling medium, i.e. air. The transfer of this heat results in enthalpy increase in air. The increase in this enthalpy may be divided into the one connected with the mass transfer and into the other connected with the convective heat transfer, i.e.:

$$
d Q=d Q_{m}+d Q_{c}
$$

Then, enthalpy change caused by mass transfer may be expressed as:

$$
d Q_{m}=i_{v} h_{d}\left(w_{s w}-w\right) d A,
$$

where $w_{S W}$ is the humidity ratio of the saturated air at the water temperature. For the enthalpy change caused by convective heat transfer, it may be written:

$$
d Q_{c}=h\left(T_{w}-T_{a}\right) d A
$$

where for the difference $T_{w}-T_{a}$, it may be written:

$$
T_{w}-T_{a}=\frac{\left[\left(i_{m a s w}-i_{m a}\right)-\left(w_{s w}-w\right) i_{v}\right]}{c_{p m a}} .
$$

\footnotetext{
${ }^{a}$ Corresponding author: L.Dvorak@fs.cvut.cz
} 
By substituting such relations into the equation (1) and with the assumption that the Lewis factor is equal to unity $\left(L e_{f}=h /\left(c_{p m a} h_{d}\right)=1\right)$ we get the relation:

$$
d Q=h_{d}\left(i_{m a s w}-i_{m a}\right) d A .
$$

The heat conveyed in the control area within the rain zone may also be expressed by calorimetric equations, thus writing the thermal balance between water and air for the control area:

$$
d Q=m_{w} c_{p w} d T_{w}=m_{a} d i_{m a}
$$

If we substitute the relation $d T_{w}=$ $d i_{\text {masw }} /\left(d i_{\text {masw }} / d T_{w}\right)$ into the left part of the equation (6) and modify the equation, we get the relation:

$$
d i_{\text {masw }}=d Q\left(d i_{\text {masw }} / d T_{w}\right) /\left(m_{w} c_{p w}\right) .
$$

If we subtract the air enthalpy change from the equation $d Q=m_{a} d i_{m a}$ and from the equation (5) from the equation (7), we get the modified relation:

$$
\frac{d\left(i_{m a s w}-i_{m a}\right)}{\left(i_{m a s w}-i_{m a}\right)}=h_{d}\left(\frac{\left(d i_{m a s w} / d T_{w}\right)}{m_{w} c_{p w}}-\frac{1}{m_{a}}\right) d A .
$$

Compare the equation (8) with the equation to derive the mean logarithmic temperature gradient of a heat exchanger:

$$
\frac{d\left(T_{h}-T_{c}\right)}{\left(T_{h}-T_{c}\right)}=-U\left(\frac{1}{m_{h} c_{p h}}-\frac{1}{m_{h} c_{p h}}\right) d A
$$

where indexes $h$ and $c$ are used for hot and cold fluid. The comparison results in the definition of the heat capacity for air (i.e. cold medium) as $m_{a}$ and the heat capacity for water (i.e. hot medium) as $\left(m_{w} c_{p w}\right) /\left(d i_{\text {masw }} / d T_{w}\right)$.

The maximum quantity of heat that can be transferred can be defined as follows:

$$
Q_{\max }=C_{\text {emin }} \cdot\left(i_{\text {maswi }}-\lambda-i_{\text {mai }}\right)
$$

where $i_{\text {maswi }}$ is the saturated air enthalpy at the water inlet temperature, $i_{\text {mai }}$ is the air inlet enthalpy and $\lambda$ is the correction factor given by equation:

$$
\lambda=\left(i_{\text {maswo }}+i_{\text {maswi }}-2 i_{\text {masw }}\right) / 4,
$$

where $i_{\text {masw }}$ is the saturated air enthalpy at the water mean temperature $T_{w m}=\left(T_{w i}+T_{w o}\right) / 2$.

Two calculation variants shown in Table 1 are considered for further calculation. From relations given in the table, the minimum heat capacity may be determined and substituted into the relation (10) and the maximum theoretical heat that can be transferred may be determined. Effectiveness of a heat exchanger - in this case, the rain zone is then given by relation:

$$
e_{e}=\frac{Q}{Q_{\max }}
$$

Table 1. Determination of the minimum heat capacity.

\begin{tabular}{|c|c|}
\hline Case "1" & Case "2" \\
\hline$C_{c}>C_{h}$ & $C_{h}>C_{c}$ \\
\hline$m_{a}>\frac{\left(m_{w} c_{p w}\right)}{\left(d i_{\text {masw }} / d T_{w}\right)}$ & $\frac{\left(m_{w} c_{p w}\right)}{\left(d i_{\text {masw }} / d T_{w}\right)}>m_{a}$ \\
\hline$C_{\text {emin }}=\frac{\left(m_{w} c_{p w}\right)}{\left(d i_{\text {masw }} / d T_{w}\right)}$ & $C_{\text {emin }}=m_{a}$ \\
\hline$C_{e}=\frac{\left(m_{w} c_{p w}\right)}{\left(d i_{\text {masw }} / d T_{w}\right) \cdot m_{a}}$ & $C_{e}=\frac{\left(d i_{\text {masw }} / d T_{w}\right) \cdot m_{a}}{\left(m_{w} c_{p w}\right)}$ \\
\hline
\end{tabular}

\section{Experimental line}

Following the above mentioned, an experimental line has been designed (Figure 1). As resulting from the configuration of the whole cooling tower, the mutual direction of the vectors of the velocity fields of individual phases (water - humid air) in the rain zone is generally in all angles and is not purely counterflow or crossflow. Therefore, the resulting mathematical model must be at least two-dimensional model. However, obtaining the accurate data for at least one direction will be sufficient to verify the mathematical model. Therefore, the "almost" crossflow model was selected as shown in schemes below. However, the ideally crossflow connection is not possible due to the drift of drops by flowing air. However, given the low measuring area $(0.5 \mathrm{~m})$, this drift of liquid phase may be neglected.

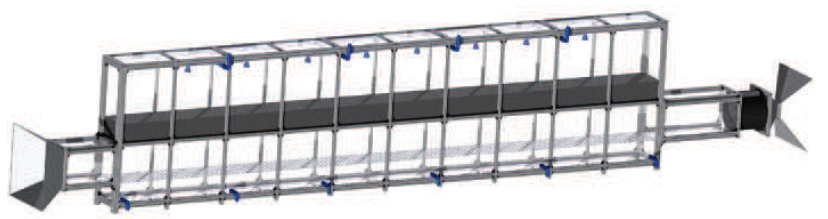

Figure 1. Experimental line

\subsection{Water system}

Water is supplied to the stand from an elevated tank equipped with heaters and overflow discharge to maintain the constant level as well as the constant flow of water through the measuring section. The constant level is maintained by means of a pump, which circulates water from one of the parts of the water tank (equipped with heaters) to the other (feeding). To increase the flow rate, it is supplied by means of two pipes (Figure 2), each equipped with an inductive flowmeter, control valve and a temperature sensor. High-pressure nozzles, with their patterns corresponding to stand width, are mounted on the supply line. Nozzles are used for distributing water to film cooling fills, with water coming out of it in the form of drops to the measuring area. Eight separate collecting vessels are located beneath the fills, which allow to monitor the change in water temperature along the entire line as well as to monitor the amount of conveyed heat in individual sections, whose lengths correspond to the 
lengths of vessels. Water is then drained in piping to the adjacent water channel from each tank.

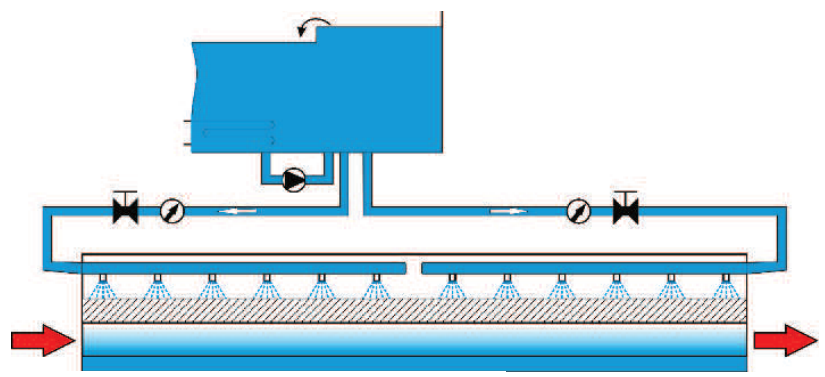

Figure 2. Water system scheme

\subsection{Air system}

The entire line is designed as exhausted by means of a two-stage axial fan. For uniform velocity profile, the inlet is equipped with a symmetric sine air-conditioning adapting piece. Due to relatively low velocities in the measuring section, a contraction in the form of so-called "two-sine nozzle" is created at the outlet [Jonáš 1991]. Four static pressure taps and two taps of total pressure with Kiel rings are located at the narrowest point of the nozzle. At this narrow point, the individual extractions are used for determining the dynamic pressure and then the average velocity of air along the rain zone. Three sensors determining the actual static pressure, temperature and air humidity are located at the inlet (in the direction of air flow after inlet nozzle) and at the outlet (before contraction).
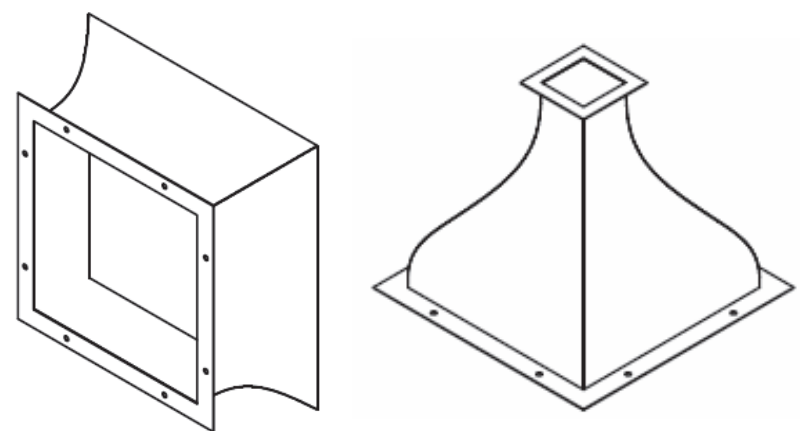

Figure 3. Left - Nozzle at the inlet to the measuring area; Right - Nozzle for measuring the air velocity

\section{Evaluation of results}

Data from all sensors are recorded throughout the measurement and parameters of both fluids - i.e. cooling and cooled, are then real-time evaluated. Given the relatively high cooling performance of the whole experimental system it is then impossible to measure continuously, however with the maximum water flow, it is possible to operate the line for approximately 30 minutes, during which the heat and mass transfer in the stand stabilises around constant values. The values obtained on the experimental stand are then used for evaluating the change in air enthalpy after passing through the actual length of measuring section. The length of measuring section can be then discreetly shortened by closing the individual nozzles or only one of two water pipes may be possibly used. The inlet temperature of each pipe and the water outlet temperature of each of the collecting vessels are then measured on the water side. An example of outlet temperatures of individual vessels is given in Figure 4. This chart shows that the most intensive heat transfer (maximum cooling of the water) occurs at the beginning of the rain zone due to the highest temperature and concentration gradient.

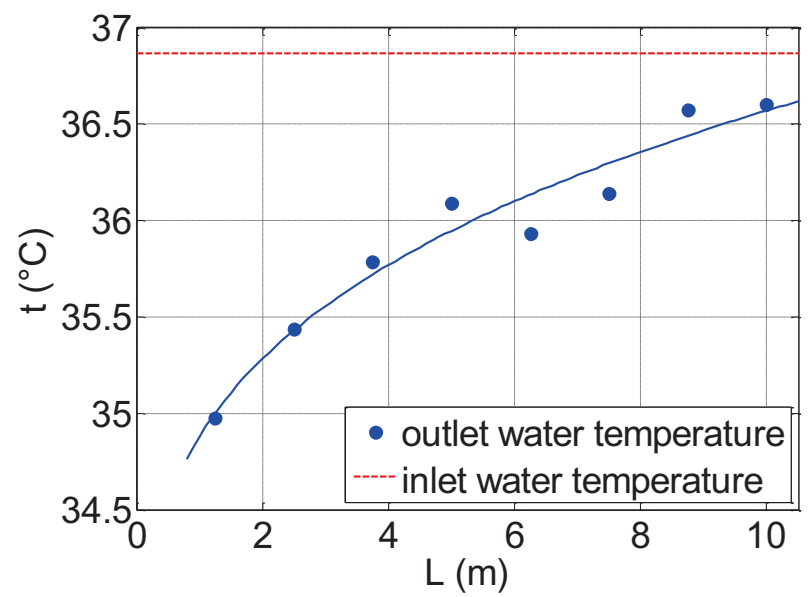

Figure 4. Course of water temperatures along experimental stand

Throughout the measurement, the total heat flux, both on the water side and on the air side, is evaluated from data obtained from individual sensors. To determine quality of the measurement it is then possible to use the heat balance defined as ratio of the heat fluxes. An example of recording the actual heat fluxes evaluated from individual meters is given in Figure 5. The record shows a very good agreement between the heat removed from the water and transferred to the air.

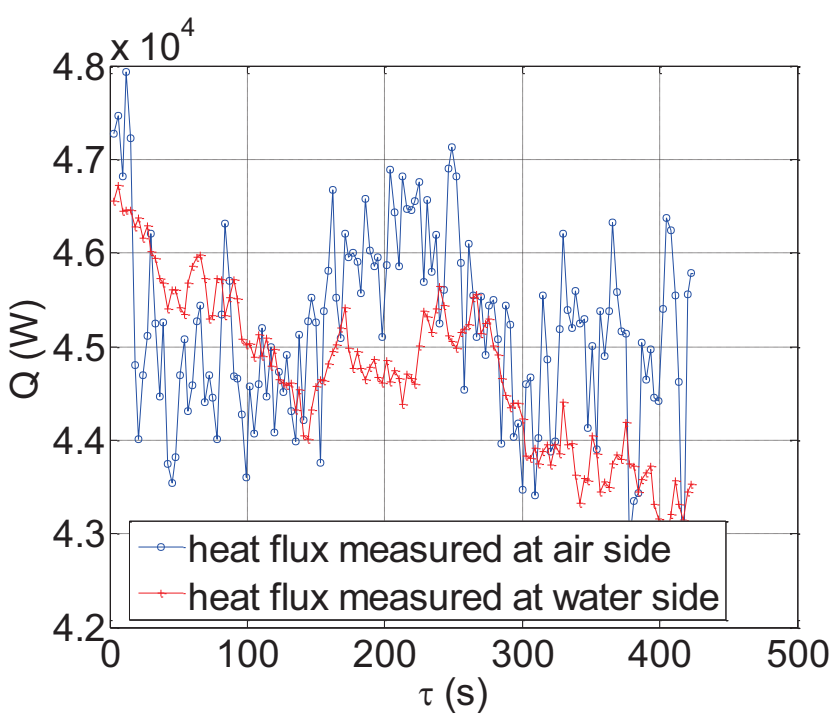

Figure 5. Time characteristic of measured heat flux

A non-dimensional characteristic Merkel number of an additive nature is used for determining the rate of heat and mass transfer in cooling towers. Therefore, it can be 
said that for individual sections of a cooling tower, in which heat and mass transfer happen, i.e. the spray zone, fill zone and the rain zone, the total Merkel number may be defined, which is the sum of Merkel numbers of the zones. As indicated above, the so-called "Chebyshev integration", which is derived for counterflow towers, is most often used for evaluating the Merkel number of the cooling fill. Therefore, the e-NTU method, modified for evaporative cooling systems, was used for this experiment setting.

The principle of the e-NTU method is to determine the effectiveness of a heat exchanger according to the equation (12). To calculate the effectiveness of a heat exchanger, Kays [3] suggested several equations for several types of heat exchangers. The equations are the function of the ratio of heat capacities and nondimensional parameter NTU (Number of Transfer Units), which is widely used for the analysis of heat exchangers. However, this case concerns a reverse task, as the effectiveness may be easily determined from the data measured. To evaluate the Merkel number for the rain zone in the crossflow connection, it is then necessary to determine first the NTU from the following equation:

$$
e_{e}=1-\exp \left[\left(\frac{N T U^{0,22}\left\{\exp \left[-C_{e} \cdot N T U^{0,78}\right]-1\right\}}{C_{e}}\right)\right]
$$

where $C_{e}$ is determined from Table 1 . To determine the NTU this equation was solved by a simple iteration method. The Merkel number for individual modes may be then easily evaluated from the following equation:

$$
M e=N T U \frac{C_{e m i n}}{m_{w}} .
$$

The results of individual measurements are given in Fig. 6 for individual values of $\dot{m}_{w} / \dot{m}_{a}$, i.e. the ratio of mass flows of water and air.

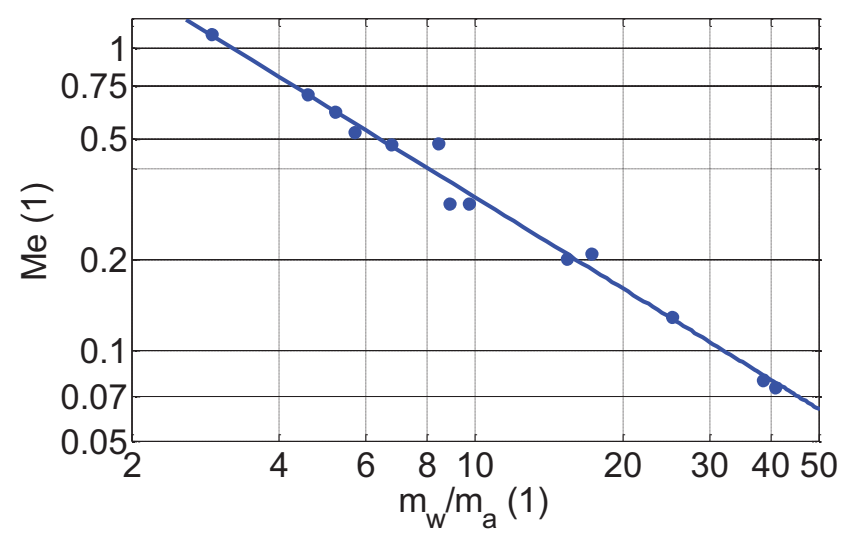

Figure 6. Dependance of the Merkel number on the ratio of mass flows of water and air

\section{Conclusion}

Within the research of the transmission of heat, momentum and mass in two-phase flow in a cooling tower, which is aimed at intensifying the performance by correct design of water spray in cooling towers, it is necessary to create a suitable mathematical model best suited to experimental data. Therefore, an experimental stand was designed and assembled to allow to obtain relevant data under appropriately defined conditions.

Data that helped in selecting and locating the suitable meters to make the measured data as accurate as possible were obtained within stand testing. The evaluated heat fluxes that were measured in individual fluids were used for calculating the heat balance of the whole experimental stand. If this balance approximates the unity, all meters may be deemed to record correct data.

For the possibility of further use of data, e.g. to verify and set the prepared mathematical model, the data obtained had to be sufficiently generalised. For this generalisation, the most frequently used characteristic was used - the socalled Merkel number. The e-NTU method that may be also applied, when modified appropriately, to evaporative cooling systems was then used for calculating the Merkel number. The Merkel numbers for individual modes were assembled in the characteristic shown in Fig. 6 depending on the actual ratio of mass flows of the two fluids.

\section{Acknowledgement}

This work has been supported by Technology Agency of the Czech Republic under the project Advanced Technologies for Heat and Electricity Production TE01020036.

Some of the measuring equipment has been purchased by Centre for research of multiphase flow and thermodynamics processes in renewable sources and energetics - NEW ENERGY reg. n. CZ.2.16/3.1.00/22130 supported by European Union.

\section{References}

1. D. G. Kröger, Air-Cooled Heat Exchangers And Cooling Towers: Thermal-Flow Performance Evaluation And Design PennWell Books, (2004)

2. H. Jaber, R. C. Webb, Journal of Heat Transfer,111, 837-843 (1989)

3. W. M. Kays, A. L. London, Compact Heat Exchangers, 3rd ed., McGraw-Hill, (1984) 